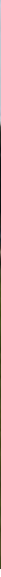

\title{
Die Entwicklung der Stadt Torgau seit 1990
}

\author{
Dagmar Rausch und Karin Hahn
}

Blick über die Elbwiesen auf die Torgauer Innenstadt - Stadtverwaltung Torgau
In Deutschland gibt es nur wenig mit Torgau vergleichbare Städte, deren historische Kontinuität und Entwicklung so deutlich im Raumgefüge ablesbar ist. Die Stadt an der Elbe blickt auf eine mehr als 1000-jährige Geschichte zurück. Torgau wurde 973 erstmals als Marktort erwähnt und war einer der großen Flussübergänge an der Elbe sowie Kreuzung wichtiger Fernhandelsstraßen. Die Stadt liegt heute im Norden des Freistaates Sachsen und ist landschaftlich in die weite Auenlandschaft der Elbe eingebettet. Über der Elbe, auf einem Porphyrfelsen, erhebt sich majestätisch das Schloss Hartenfels, hervorgegangen aus einer mittelalterlichen Burganlage. 1267 erfolgte die erste urkundliche Erwähnung als „civitas“ (Stadt).

Anfang des 16. Jahrhunderts erlangte Torgau Residenzfunktion und entwickelte sich zum politischen Zentrum Sachsens und der lutherischen Reformation. Die Stadt spielte damit eine herausragende Rolle in der Weltgeschichte, was heute selbst in Sachsen nicht allseits bekannt ist.

Gelegen am westlichen Ufer der Elbe, hatte Torgau im 19. Jahrhundert eine strategische Bedeutung als Festung, was mit drastischen Eingriffen in die Stadtstruktur verbunden war. Neben den Vorstädten waren auch große Teile der mittelalterlichen Stadtmauer betroffen. 1815 fiel Torgau an die Preußen, die Festung wurde weiter ausgebaut und ab 1890 geschliffen. Infolgedessen ging die frühe industrielle Entwicklung des 19. Jahrhunderts an Torgau vorbei. Erst mit Beginn des 20. Jahrhunderts entwickelte sich die Stadt über die Grenzen der mittelalterlichen Anlagen hinaus. Bis 1945 war in der Stadt keine rege Bautätigkeit zu spüren.
Im Zweiten Weltkrieg war die Stadt unzerstört geblieben. Die geschlossen erhaltene Altstadt konnte so zu einem Paradebeispiel für eine analytische Arbeit der Denkmalpflege werden. Nach dem Krieg wollten die Torgauer die Attraktivität des Schlosses wieder hervorheben. Es entstand im Rahmen eines Aufbauwerkes ein weitläufiger Rosengarten und der aus dem 16. Jahrhundert stammende Bärenzwinger beidseits der Zugangsbrücke wurde wieder mit Bären bestückt. 1965 veranlasste der damalige Rat des Kreises Torgau die Erneuerung der Außenanlagen von Schloss Hartenfels. Das Schlossensemble mit erneuerten Brücken, Gräben und gestalteten Freiräumen war wieder ein Kleinod geworden.

Außergewöhnlich für eine Stadt dieser Größenordnung in der DDR war auch die komplexe Herangehensweise der Erhaltung der Altstadt in den 1970 Jahren, um den weiteren Verfall zu stoppen. Die Torgauer begannen bereits 1978 mit den damaligen Möglichkeiten und mit Engagement des damaligen Institutes für Denkmalpflege, ihre Altstadt zu sanieren. Eine Planungsgruppe Städtebau wurde eingerichtet, eine professionelle Farbgestaltung für gesamte Straßenzüge vorgenommen, die Planung mit denkmalpflegerischen Zielstellungen abgestimmt und die Realisierung unter Leitung einer Koordinierungsgruppe forciert. Bis 1990 wurde in jedem Jahr ein Straßenzug saniert, mehr als 1.000 Wohnungen modernisiert und etwa 200 Läden und weitere Unterlagerungen hergerichtet.

Der pragmatische Umgang mit der Problematik Denkmalpflege, der die Anpassung der Substanz an geänderte Aufgaben und Funktionen unter 
Wahrung ihrer Wertigkeit zum Ziel hatte, war ein wesentliches Element im Umgang mit der Bausubstanz in Torgau bis 1990. Dabei spielte die Stadt als Ganzes bald eine große Rolle. Es stand und steht nicht das einzelne Objekt im Vordergrund, sondern die Altstadt in ihrer Geschlossenheit als städtebauliches Ensemble. Das Stadtbild wurde mit diesen Baumaßnahmen sehr deutlich aufgewertet und Torgau wurde zum beliebten Besucherziel nicht nur für Baufachleute. Gegenüber den meisten ostdeutschen Altstädten, die sich 1990 in einem bedauernswerten Zustand befanden, stellte die Torgauer Altstadt somit eine Ausnahme dar. Mit der Wende standen die vorhandenen Instrumentarien der Stadtentwicklung auf dem Prüfstand. Die Stadträte sowie die Stadtverwaltung hatten die Aufgabe, die neuen Bedingungen zu nutzen und Grundlagen für die notwendige weitere Entwicklung zu schaffen. Eine vollständig geänderte Rechtslage, der zunächst außerordentlich große Planungsspielraum der Kommunen, die Lösung ungeklärter Eigentumsfragen, die Reprivatisierung von Immobilien und die Erneuerung des Bodenmarktes hatten entsprechende Auswirkungen.

Der historische Wert der Altstadt war und ist mit einer Vielzahl von Problemen konfrontiert. Viel Unterstützung bekam die Stadt Torgau in dieser Zeit durch die bereits seit mehreren Jahren bestehenden guten Beziehungen zur Partnerstadt Sindelfingen in Baden-Württemberg. So konnten Weichenstellungen vorgenommen werden, die die Fortsetzung der Erhaltungs- und Gestaltungsmaßnahmen in der Altstadt auf breiterer Basis ermöglichten. Im Rückblick können wir konstatieren, dass diese Hilfe von sehr großer Bedeutung für unsere Stadt war.

\section{Einwohnerentwicklung und} demographischer Wandel

Torgau hatte 1989 mit fast 23.000 Einwohnern die bisher höchste Bevölkerungszahl erreicht. Allerdings war dieses Bevölkerungswachstum eng mit dem Ausbau des größten Industriebetriebes der Stadt, dem Flachglaskombinat, verbunden. Der 1990 einsetzende Deindustrialisierungsprozess bedingte unter anderem, dass die Einwohnerzahlen in den kommenden Jahren deutlich sanken. 1995 hatte Torgau etwa 20.300 Einwohner, 2008 waren es dann im Stadtgebiet nur noch 17.600 Einwohner. Eine hohe Arbeitslosigkeit und eine stagnierende Wirtschaftsentwicklung bewirkten einen spürbaren Abwanderungsprozess. Die Ost-WestMigration betraf insbesondere junge, qualifizierte, meist weibliche Bevölkerungsgruppen, was auch stark rückläufige Geburtenraten zur Folge hatte. Aus den rückläufigen Einwohnerzahlen bei gleichzeitig immensem Wohnungsneubau resultierten Wohnungsüberschüsse und -leerstände. Bis 1994 waren auch Suburbanisierungsprozesse durch Abwanderung ins dörfliche Umfeld zu verzeichnen. Dieser Prozess konnte in den letzten Jahren umgekehrt werden.

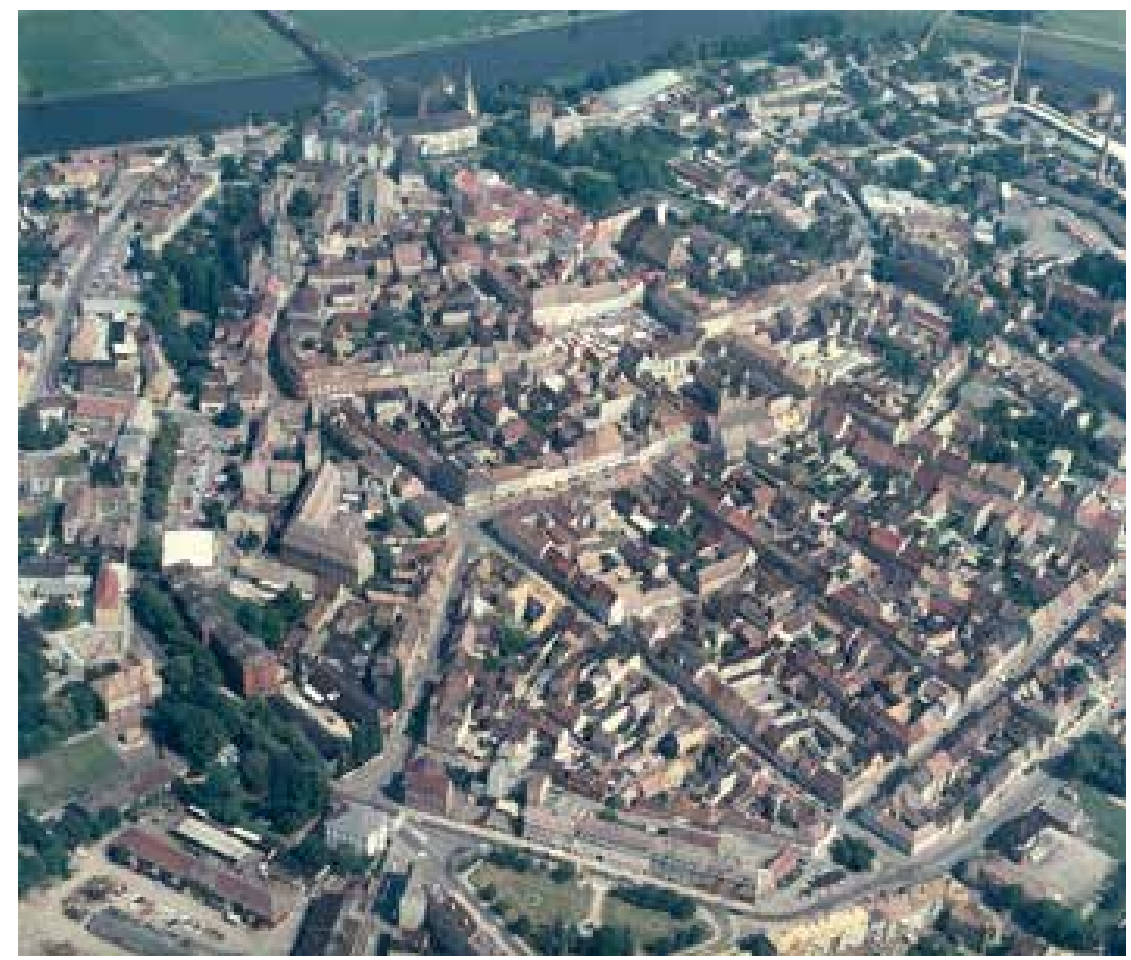

In Torgau leben heute einschließlich der eingemeindeten, früher eigenständigen Gemeinden Melpitz und Graditz (1994), Pflückuff (2009) und Zinna (2013) wieder 20.883 Einwohner. Entsprechend der Bevölkerungsprognose des Statistischen Landesamtes ist bis 2030 von einem weiteren Bevölkerungsverlust auszugehen. Die beste Variante bei der Prognose geht im Betrachtungszeitraum bis 2030 von einem Bevölkerungsrückgang von rund 1.273 Einwohnern im Vergleich zu 2015 aus. Dies entspricht einem prozentualen Verlust von etwa 6,4 Prozent. Die Geburtenzahlen liegen in Torgau im Jahr 2015 bei 46,6 Prozent gegenüber 1990 (100 Prozent). Sie werden, unter anderem aufgrund der geringen Anzahl junger Frauen, auch in Zukunft eher abnehmen.

Luftaufnahme der Torgauer Innenstadt, 1992

๑ die STEG Stuttgart/Dresden

Einwohnerentwicklung in Torgau von 1834 bis 2017

Grafik: Uwe Ulrich Jäsche

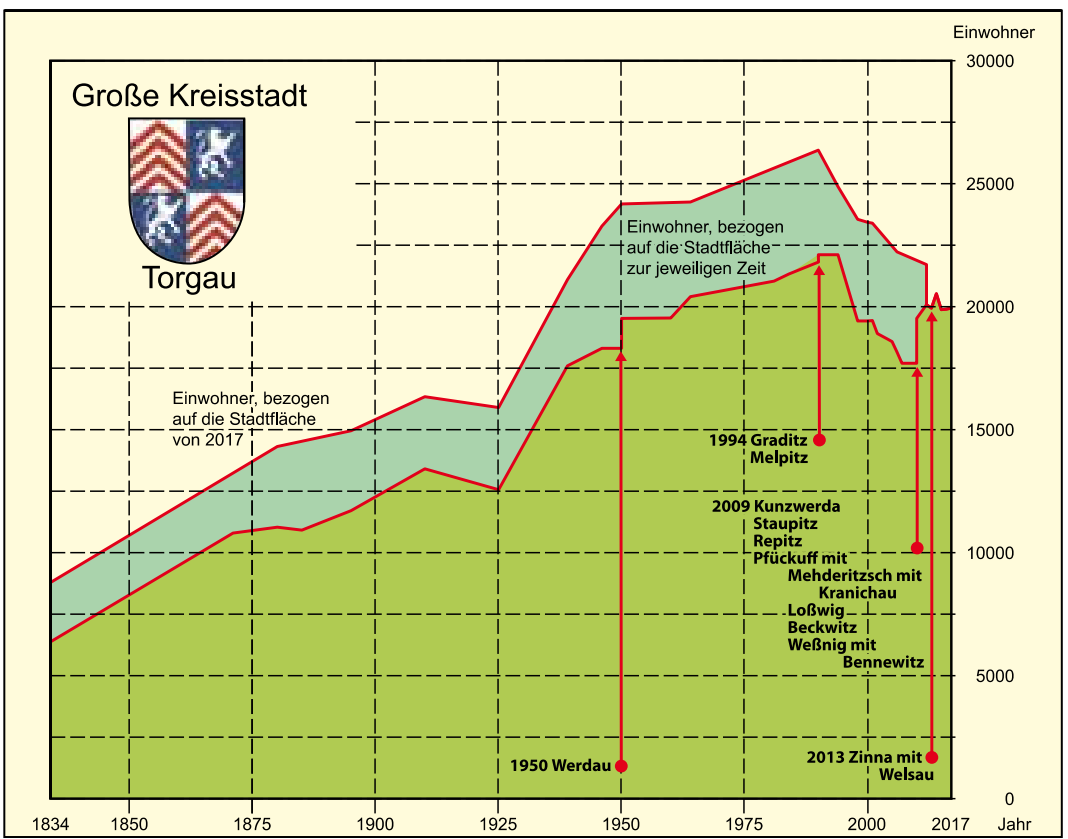


oben: Torgau, Straße der Jugend und Turnhalle Wasserturmplatz - Stadtverwaltung Torgau Husarenpark an der Dommitzscher Straße - Stadtverwaltung Torgau unten: Torgau, Wohngebiet

Der Anteil der Altersgruppe der Erwerbstätigen an der Gesamtbevölkerung nimmt tendenziell ab. Das Durchschnittsalter der Erwerbstätigen steigt dagegen immer weiter an. Junge Berufstätige sind eine der Bevölkerungsgruppen, die perspektivisch am ehesten zu einer Stabilisierung der Einwohnerentwicklung beitragen könnten. Daher ist es für die zukünftige wirtschaftliche Entwicklung wichtig, jüngere Bevölkerungsgruppen in Torgau zu halten und ihnen attraktive Arbeits- und Lebensbedingungen zu bieten.

\section{Integrierte Stadtentwicklung}

Das Integrierte Stadtentwicklungskonzept (INSEK) ist für die Stadt gegenwärtig das wichtigste Instrument zur Bewältigung der Herausforderungen des demographischen Wandels. Kernzielstellung ist „Innen- vor Außenentwicklung“. Es gilt, alternative Entwicklungsmöglichkeiten - wie das einmalige Stadtbild, die Stadtgeschichte und die natürlichen Ressourcen - optimal zu nutzen. Ziele sind insbesondere die Aufwertung und Revitalisierung der Innenstadt, die Konzentration der Sanierungstätigkeit auf den innerstädtischen und städtebaulich relevanten Altbaubestand, eine behutsame Nachverdichtung und die bedarfsgerechte Schaffung ausreichender Bauplätze.
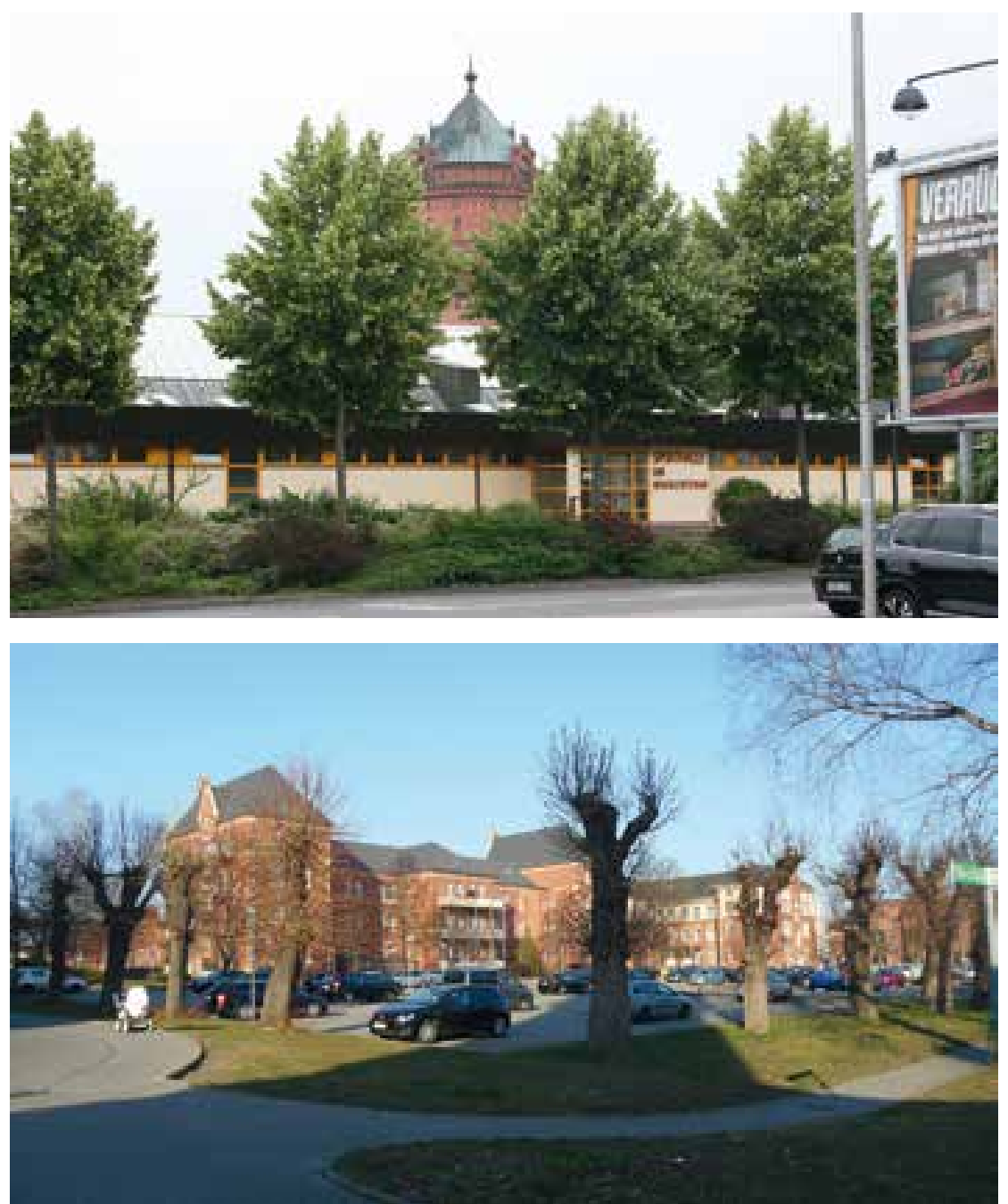

Die Stadt erarbeitete 2010 unter Einbeziehung örtlicher Akteure Entwicklungsstrategien für viele Bereiche des öffentlichen Lebens und aktualisierte daraus abgeleitet das Stadtentwicklungskonzept. Es umfasst, aufbauend auf umfangreichen Analysen, Aussagen zur Bevölkerungsentwicklung und zur gesamtstädtischen Entwicklung sowie Fachkonzepte zu Themen wie beispielsweise Städtebau und Denkmalpflege, Wohnen, Soziales. Diese Zielstellungen sind weiter zu präzisieren und fortzuschreiben.

\section{Wohnungsbau, Wirtschaft, Infrastruktur}

In der ersten Hälfte der 1990er Jahre stand die Städtebaupolitik in Deutschland noch unter dem Primat von Wachstumsoptionen. Die Folge dieses Prozesses im näheren und weiteren Umfeld war, dass neben Einkaufszentren neue Gewerbegebiete und Eigenheimsiedlungen entstanden, die unter anderem eine erhebliche Ausweitung der bebauten Flächen nicht nur am Stadtrand, sondern auch in den angrenzenden Dörfern nach sich zogen. Die Stadt Torgau hat eine Stadtentwicklungspolitik betrieben, die diesem Trend nur gemäßigt folgte. Es wurden neue Gewerbe-, Handels- und Wohnbauflächen ausgewiesen, diese aber stufenweise und bedarfsorientiert entwickelt. Größere Neubauten, wie 1993 eine neue Turnhalle am Wasserturmplatz und die Grundschule am Rodelberg, konnten dennoch im Innenbereich schnell realisiert werden.

Die Bautätigkeit in der Stadt Torgau konzentrierte sich nach 1990 klar auf die Sanierung des Bestandes bei vergleichsweise geringfügigen Zuwächsen in den Wohnungsbestandszahlen. Im Wohnungsneubau hatte sich Torgau auf den Einfamilienhausbau konzentriert, nicht auf den Geschosswohnungsbau. Diesem Sachverhalt ist es zu verdanken, dass in Torgau nach der Wende nicht am Wohnungsmarkt vorbei gebaut wurde. Aus städtebaulicher Sicht entsprach diese Vorgehensweise damit weitgehend den objektiv begründeten Reproduktionserfordernissen.

Trotz des quantitativ ausreichenden Wohnungsangebotes führten unter anderem die niedrige Eigentumsquote und die Wohnbedürfnisse der Bevölkerung dennoch nach 1990 zur Entwicklung von mehreren neuen Baugebieten mit Bauflächen für den Eigenheimbau. So entstanden in den letzten Jahren Gebiete mit insgesamt etwa 240 Eigenheimgrundstücken unter anderem in der Nähe des Großen Teiches in landschaftlich attraktiver Lage, an der Thomas-Müntzer-Straße sowie im Wohngebiet Eilenburger Straße auf einer Rückbaufläche mit guter Erreichbarkeit des Stadtzentrums. Des Weiteren ist ein attraktiver Wohnstandort ab 1998 auf einer Konversionsfläche an der Dommitzscher Straße entstanden. Die drei Unterkunftsgebäude im Gelände der ehemaligen Husarenkaserne wurden zu 94 modernen Wohneinheiten umgebaut. Im Inneren des Geländes wurden 45 Reihenhäuser ausgebaut.

Bei der Fortschreibung des INSEK wurde der Leerstand neu erfasst, und es wurde deutlich, dass er durch den Stadtumbau signifikant gesenkt werden 
konnte. Der Wohnungsmarkt konnte in den letzten Jahren quantitativ weitgehend den Anforderungen angepasst werden. Im Fokus zukünftiger Betrachtungen dürften besonders die Altstadt und weiter auch Torgau-Nordwest liegen. Planmäßig soll die Bebauungsdichte zum Stadtrand hin abnehmen.

Die Stadt muss sich zudem stärker auf die zunehmende Zahl älterer Bürger einstellen und Anpassungsmaßnahmen zum Beispiel hinsichtlich eines altengerechten Wohnungsangebotes, einer altengerechten Stadtgestaltung (Barrierefreiheit) und vielfältigeren Angeboten für Senioren vornehmen. Die Prognose zeigt einen deutlichen Anstieg der Jahrgänge über 65 Jahre auf bis zu 34 Prozent im Jahr 2030. Die große Nachfrage nach geeigneten Wohnformen für Ältere kann derzeit noch nicht ausreichend befriedigt werden. Es gibt einige gute Beispiele, wie das Senioren-Selbsthilfe-Zentrum im Fleischmarkt und am Altstadtrand, den Neubau von 25 altersgerechten Wohnungen in der Schlachthofstraße, den Umbau von 64 barrierearmen Wohnungen am Altstadtrand in der Lassallestraße oder die Sanierung von 33 Wohneinheiten in der Puschkinstraße, zum Teil mit diversen Serviceangeboten.

Neben dem Wohnen sind Handel, Dienstleistungen sowie Kultur und Bildung wesentliche Bausteine der Stadtentwicklung.

Ungünstige Standortfaktoren wie die periphere Lage der Stadt, das Vorhandensein einer Vielzahl von naturräumlichen Restriktionen (Naturschutzgebiete, Hochwasserschutz, Trinkwasserschutz) und die ungenügende Verkehrsanbindung schränkten jedoch die Geschwindigkeit, kurzfristig neues Gewerbe und Industrie anzusiedeln, stark ein. Torgau war vor 1990 Sitz des führenden Industriekombinats in der Flachglasherstellung. Dort wurden ca. 80 Prozent der gesamten Flachglasproduktion der DDR erzeugt. Seit 1926 ist in Torgau die Porzellan- und Steingutproduktion zu Hause und hatte auch als Standort der Möbelproduktion, des Landmaschinenbaus und der Schuhherstellung Bedeutung. Inzwischen wurden die Glasindustrie Torgau durch Saint Gobain Glass Deutschland und die Steingutfabrikation im Rahmen der Besitzrückführung von der Villeroy \& Boch AG übernommen. Durch diese Entwicklung konnte ein Teil der industriellen Produktionskapazität in Torgau erhalten werden. Weiterhin konnten in den letzten Jahren die Solartechnik und die Holzindustrie als ergänzende Wirtschaftsfelder erfolgreich platziert werden. Torgau als zweitgrößter Waldbesitzer in Sachsen (1.200 Hektar Stadtwald) hat enorme Ressourcen in der Holzwirtschaft vor Ort. Aktuell müssen einige Industriebrachen dringend einer Beräumung und anschließenden Revitalisierung zugeführt werden. Diese sollen aufgrund ihrer überwiegend innerörtlichen Lage vorrangig der Verdichtung des Innenbereiches dienen. Für die im Außenbereich befindlichen Flächen wird eine Renaturierung angestrebt.

Insgesamt konnte sich die Torgauer Wirtschaft nach den Umbrüchen durch den Strukturwandel

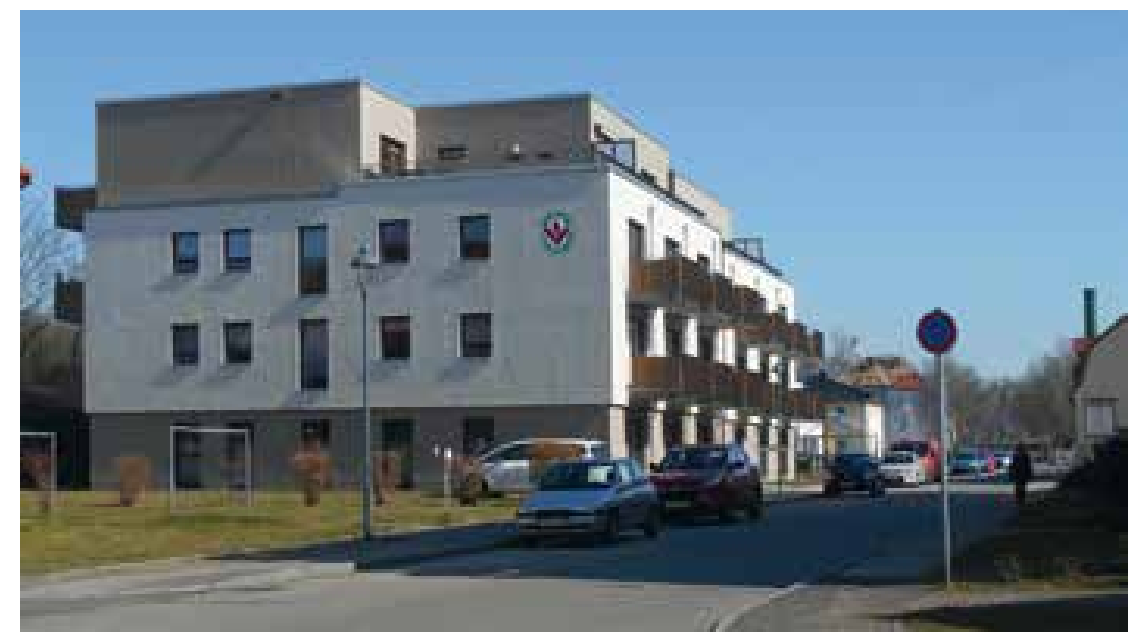

stabilisieren. Ein wichtiger und für die Stadtentwicklung ebenfalls sehr bedeutender Wirtschaftszweig ist der der öffentlichen und privaten Dienstleister.

Von gehobener Bedeutung für die Stadtentwicklung ist weiterhin die Verbesserung der überregionalen Verkehrsanbindung. Der 1993 dringend notwendig gewordene Bau der neuen Elbbrücke hatte zu heftigen Diskussionen in der Bevölkerung geführt. Die Planungen des Freistaates beziehen sich neben Netzverbesserungen im Süden der Stadt im Wesentlichen auf die B 87n, die aufgrund des Fehlens einer Autobahnanbindung große Bedeutung für die Region Torgau als Anschluss an den Ballungsraum Leipzig und nach Osten an den Raum Frankfurt/Oder, Cottbus und Polen hat. Gemeinsame Anstrengungen der gesamten Region, der Kommunen, der Wirtschaft sind nötig, um dieses Vorhaben nach nunmehr fast 30 Jahren Diskussion und unterschiedlicher Planungen endlich realisieren zu können.

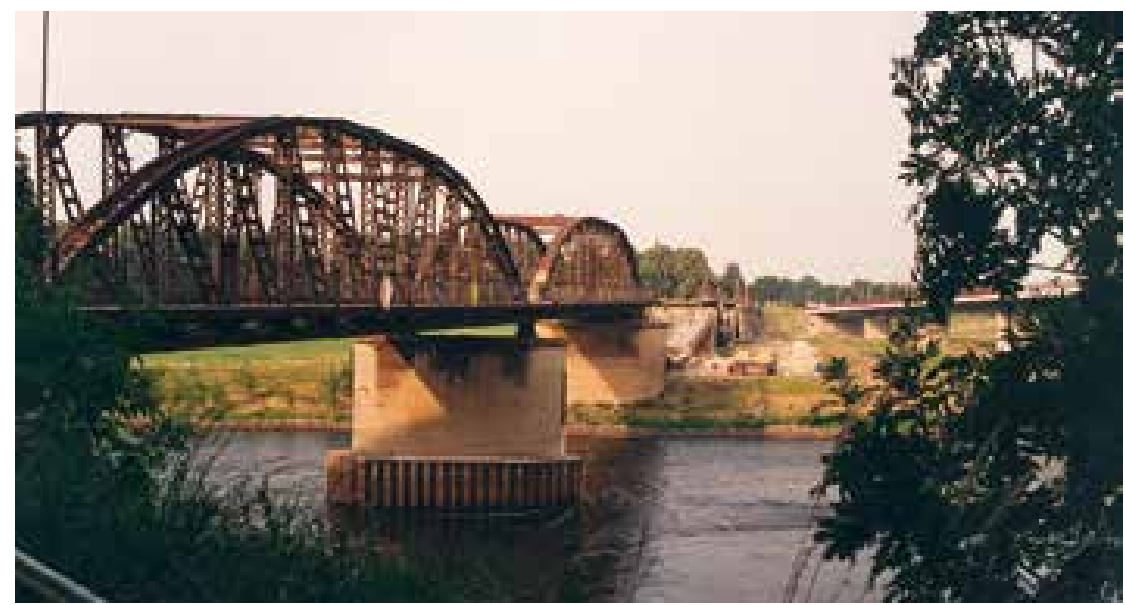

Mit der Wiedereinführung der Länder entschied sich der ehemalige Kreis Torgau nach einem regionalen Volksentscheid für den Freistaat Sachsen. Torgau ist Verwaltungssitz des seit 2008 bestehenden, sehr großen Landkreises Nordsachsen und hat eine lange Tradition als Versorgungs-, Verwaltungsund Dienstleistungszentrum. Die landesplanerischen Ausweisungen als Mittelzentrum und als Ziel des Städtetourismus sollen das unterstreichen.
Torgau, Schlachthofstraße, Neubau der Volkssolidarität mit altengerechtem Wohnen, 2014 o Stadtverwaltung Torgau
Alte Elbbrücke, rechts Bau der neuen Elbbrücke, 1992 ๑ Stadtverwaltung Torgau 
Dennoch ist dies nicht ausreichend, wenn eine der wichtigsten gesellschaftspolitischen Zukunftsaufgaben, die Herstellung gleichwertiger Lebensverhältnisse in ganz Deutschland, ernsthaft betrieben werden soll. Eine bürgernahe Verwaltung, gut erreichbare hochwertige Bildungsangebote, eine umfassende medizinische Versorgung und insgesamt eine gute infrastrukturelle Ausstattung und eben auch eine gute Erreichbarkeit sind grundlegend für die Realisierung dieses politischen Ziels.

\section{Stadtumbau und Städtebauförderung}

Die Stadt gliedert sich räumlich hauptsächlich in drei Stadtgebiete: die historische Altstadt, das Wohngebiet Eilenburger Straße (entstanden um 1960) und das Neubaugebiet Torgau-Nordwest (entstanden ab 1980). Hinzu treten die Industrie- und Gewerbegebiete, vorwiegend im Norden angesiedelt.

Die Altstadt hat eine Größe von mehr als 160 Hektar. Die überwiegend geschlossenen Quartiere sind gekennzeichnet durch eine hohe gestalterische Qualität und größtenteils Denkmaleigenschaft, welche oft auch eine Hof- bzw. rückwärtige Bebauung mit einschließen. Hier befinden sich zahlreiche baugeschichtlich wertvolle Einzeldenkmale, vorwiegend der Renaissance aber auch der Spätgotik und des Barock. Bedeutende Einzeldenkmale sind das Schloss Hartenfels, die Stadtkirche St. Marien, die ehemalige Kirche St. Nikolai, das Renaissance-Rathaus, die alte Kursächsische Kanzlei und repräsentative Bürgerhäuser des 16. und 17. Jahrhunderts. Der Altstadtkern wird wieder- um von zwei-, zum Teil auch drei- und mehrgeschossigen Gebäuden mit qualitätvollem Villencharakter und Hauszeilen umringt. Umgeben wird dieser historische Bereich vom Stadtpark, der das Vorfeld der geschliffenen und bis heute noch ablesbaren sternförmigen Festungsanlage darstellt und nach Osten hin in die offene Elblandschaft übergeht. Zu den denkmalgeschützten Bereichen gehören auch die Reste der Festungsanlage, etwa der Brückenkopf oder Repitz.

Das Luftbild zeigt deutlich die über Jahrhunderte erhaltene Stadtstruktur. Als städtebauliches Ensemble verfügt Torgau über eine besondere Wertigkeit des Raumgefüges in der historischen Altstadt. Die Anordnung der Baukörper und die klare Struktur der Platz- und Straßenräume sind eine deutliche Widerspiegelung der historischen Entwicklung der Stadt und ihrer Wachstumsphasen. Die aktuelle Liste der Kulturdenkmale Torgaus umfasst neben ca. 710 Einzeldenkmalen, von denen sich 500 im Stadtkern befinden, auch mehrere Sachgesamtheiten. Des Weiteren ist der Altstadtkern seit 2015 als Gebietssatzung unter Schutz gestellt. Ein einziger Blick auf die Denkmalkarte der Stadt Torgau veranschaulicht die Fülle der sich daraus ergebenden Aufgaben.

Das Stadtgebiet Eilenburger Straße besitzt einen hohen Bestand an Wohnungen in Gebäuden der 1960er Jahre. Dadurch fehlt die Vielfältigkeit an differenzierten Wohnungsgrößen. Der Bestand wurde qualitativ aufgewertet, es wurden Quartiersanierungen und Wohnumfeldverbesserungen vorgenommen und ein breiteres Angebot für

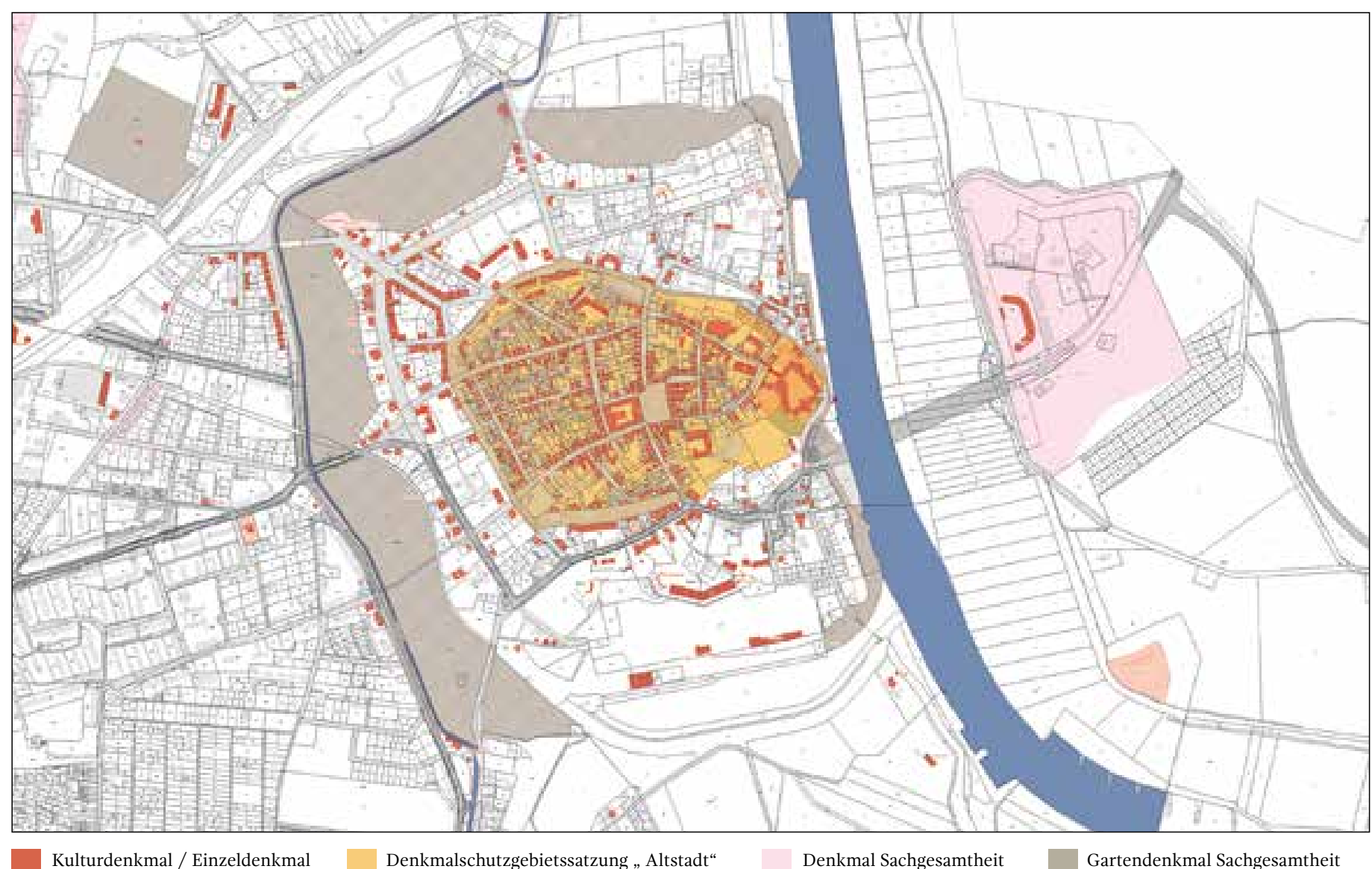




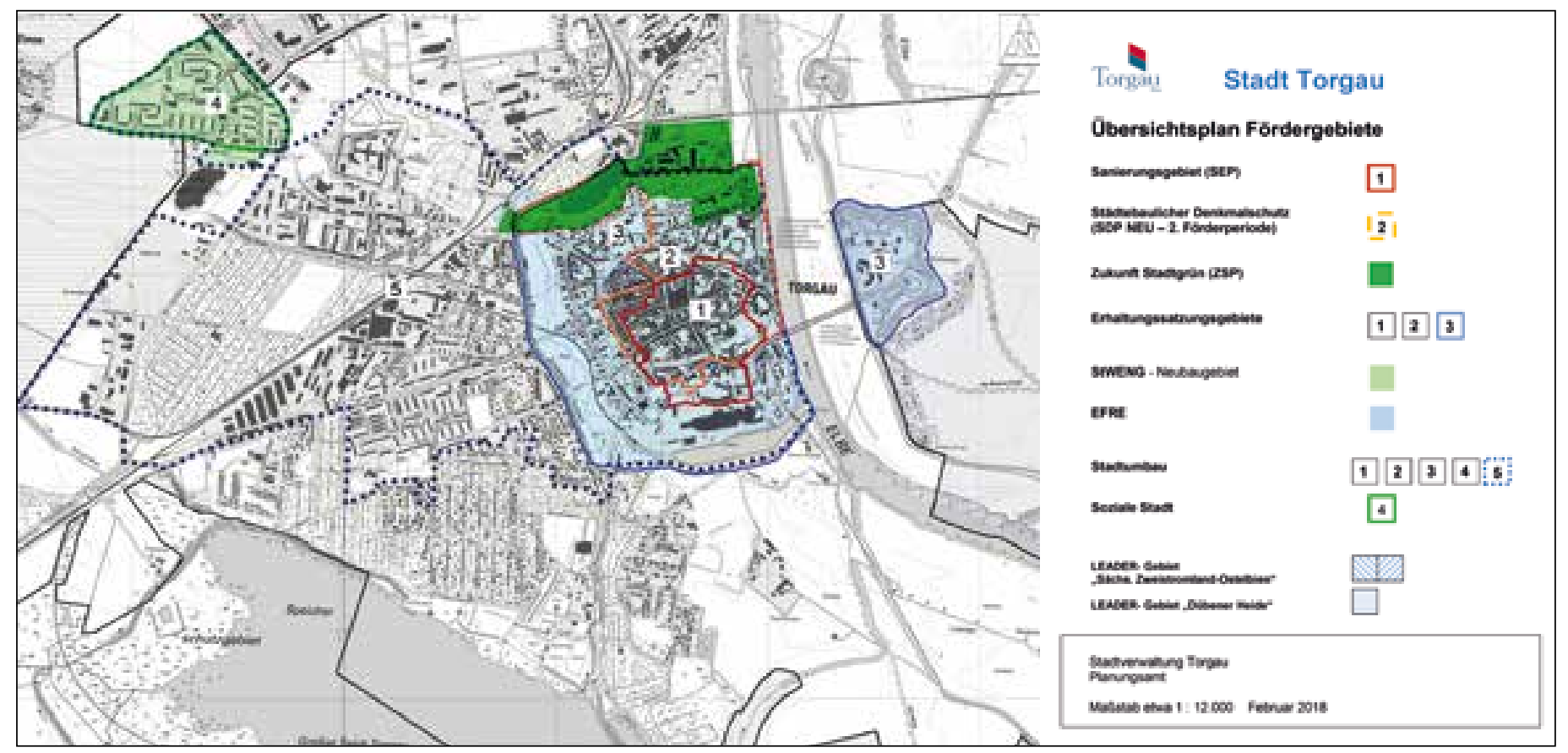

unterschiedliche Bedürfnisse geschaffen. Das Gebiet ist auch heute ein beliebtes Wohnquartier in der Stadt und nicht von einer Leerstandsproblematik betroffen. Ergänzende Planungen für Eigenheimgebiete wurden realisiert und vervollständigen die soziale Durchmischung. Infrastruktureinrichtungen wie Kitas, Schulen und Handel sorgen für ein umfassendes Versorgungssystem.

Das Stadtgebiet Torgau-Nordwest besteht überwiegend aus den Bauten der 1980er Jahre. Optisch präsentiert sich das Gebiet in einem guten $\mathrm{Zu}$ stand, was auf die Realisierung vielfältiger Städtebauprogramme zurückzuführen ist. Dennoch war hier der Einwohnerrückgang am höchsten. Die Lage des Stadtgebietes, am weitesten vom Stadtzentrum entfernt, hat diesen Prozess begünstigt.

Vor dem Hintergrund von steigenden Wohnungsleerständen kam es Ende der 1990er Jahre in der Stadtentwicklungspolitik zu einem Umdenken. Zuvor wurde Stadtentwicklung mit ständigem Wachs- tum an bebauter Fläche übersetzt. Stadtumbau bezeichnet nun eine Entwicklungsstrategie, die den Rückbau dauerhaft leer stehender und langfristig nicht mehr benötigter Wohnungen sowie von Anlagen und Netzen der sozialen und technischen Infrastruktur beinhaltet.

Die Städtebauförderung spielt bei der Entwicklung der Stadt Torgau eine große Rolle. Bereits seit 1991 werden im Rahmen der Städtebauförderung umfangreiche finanzielle Mittel des Bundes und des Freistaates in der Altstadt und im Stadtgebiet Torgau-Nordwest eingesetzt. Die öffentlichen Vorhaben dienen hauptsächlich der Verbesserung der sozialen und kulturellen sowie der Aufwertung der verkehrstechnischen Infrastruktur. Insgesamt wurden für die Sanierung in den sechs verschiedenen Fördergebieten der Stadt bereits mehr als 80 Millionen Euro investiert.

Auf den fortlaufenden Bevölkerungsverlust und die daraus erwachsende Gefahr des sich verfestigenden Wohnungsleerstandes wurde in Torgau

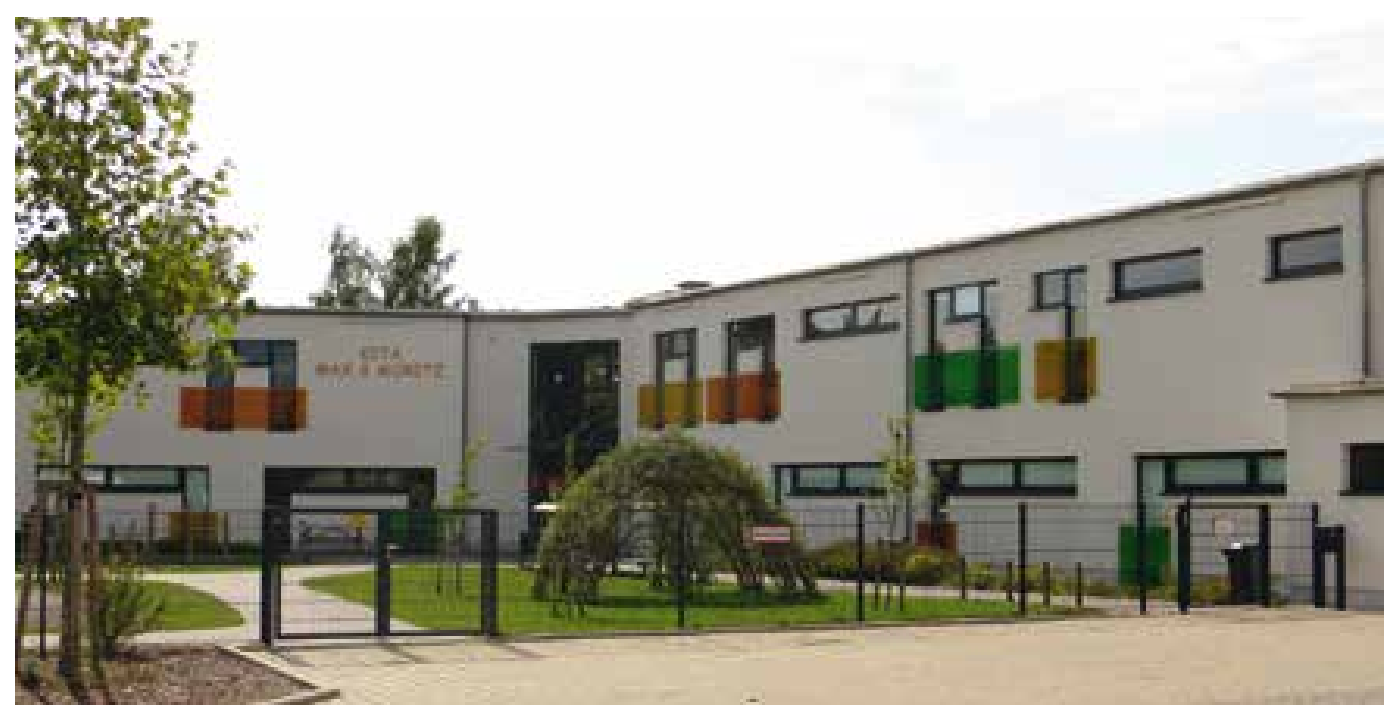

Übersicht über die

Fördergebiete in Torgau

- Stadtverwaltung Torgau
Neubau der Kindertagesstätte Max \& Moritz

๑) Stadtverwaltung Torgau 


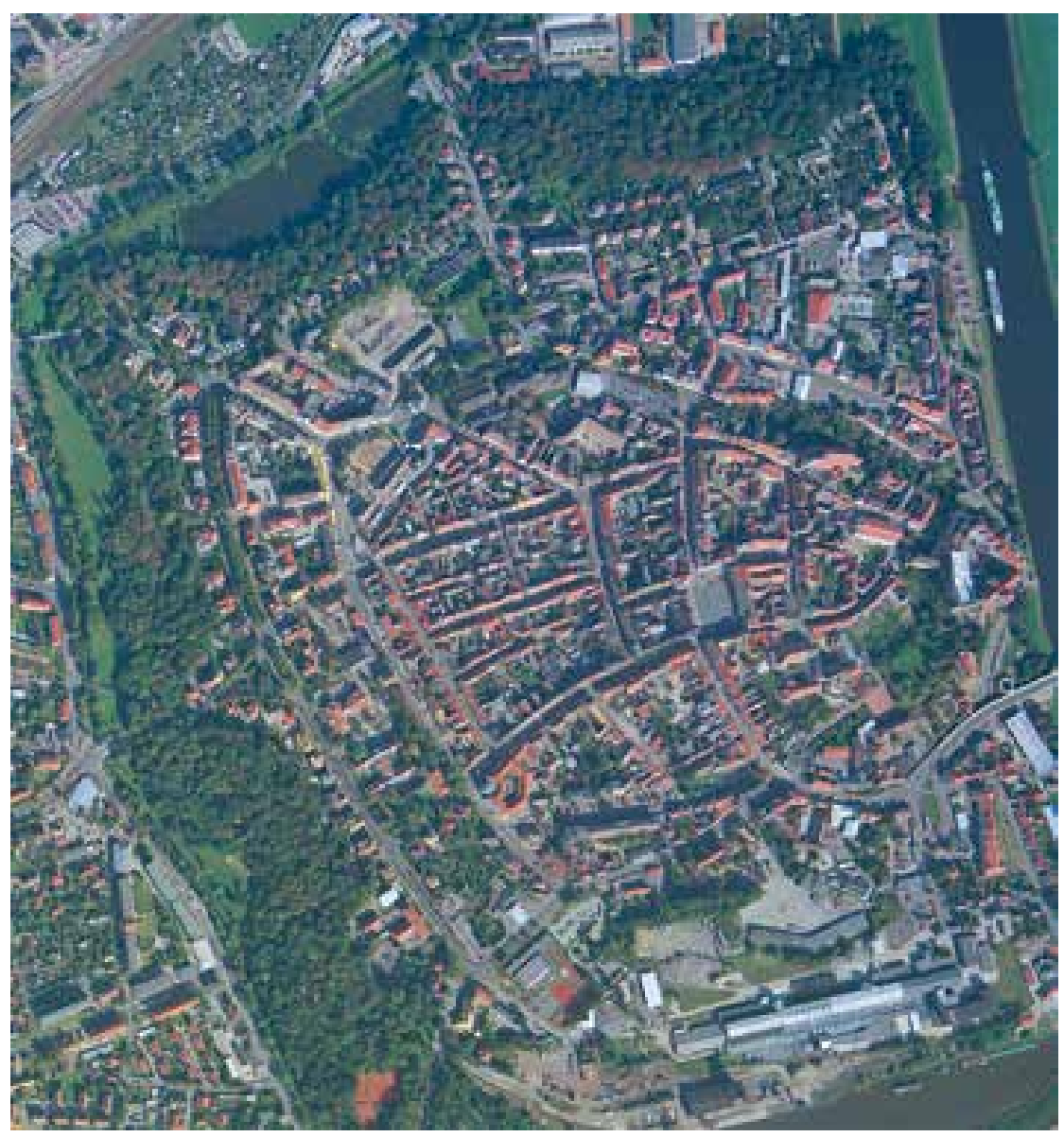

Luftbild der Torgauer Innenstadt, 2000 ๑ Stadtverwaltung Torgau

Torgau, Soziokulturelles Zentrum Kulturbastion in einer sanierten Festungsbastion ๑ Stadtverwaltung Torgau

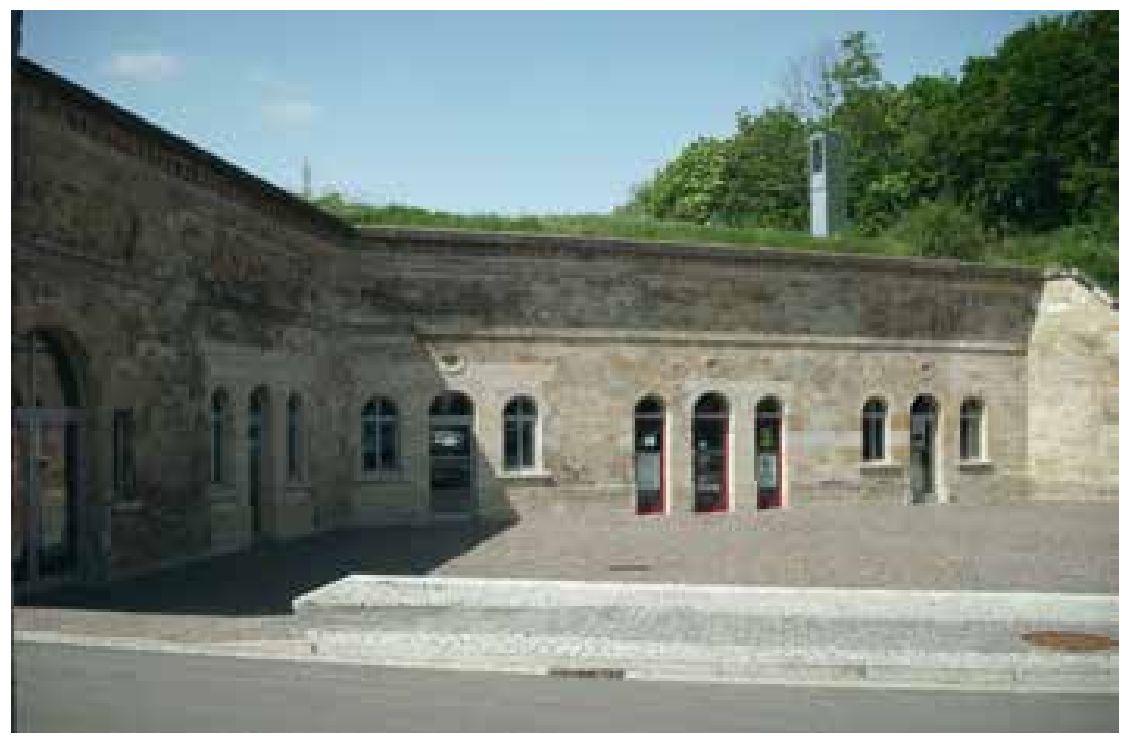

gute Ausstattung mit Schulen, Kitas, medizinischen Einrichtungen und umfassenden Handel bei jungen Familien beliebt ist.

Durch die periphere Lage im Raum mit weiten Distanzen zu den nächsten konkurrierenden Zentren wie auch durch das attraktive Stadtbild bestehen in Torgau im Prinzip gute Voraussetzungen, diese Qualitäten zu erhalten und als Motor für die zukünftige Entwicklung der Stadt zu nutzen.

\section{Innenstadtentwicklung}

Um die Attraktivität der Innenstadt zu erhalten, wurden in den vergangenen Jahren verschiedene Projekte initiiert. Beispielhaft war die Umsetzung der im Rahmen des Programms „Europäischer Fonds für regionale Entwicklung“ (EFRE) laufenden Projekte der Bildung, Kultur, Wirtschaft und des Sports in den Jahren 2004 bis 2008. Dazu hatte Torgau drei Hauptentwicklungsrichtungen gewählt. Diese waren die Unterstützung von Wirtschaft und Tourismus, von freizeitbezogener und soziokultureller Infrastruktur und von Maßnahmen der städtebaulichen Sanierung, die untereinander abgestimmt und verbunden wurden und die Synergieeffekte erzeugten.

So wurde beispielsweise das Projekt „Museumslandschaft" entwickelt, das neben dem Ausbau der Bausubstanz auch eine inhaltliche und räumliche Vernetzung dieser Angebote untereinander in der Altstadt von Torgau organisierte. Eines der tragenden Projekte dieser Maßnahme war neben der Ausstattung des Stadt- und Kulturgeschichtlichen Museums im Kanzleihaus die Sanierung des prunkvollen Bürgermeister-Ringenhain-Hauses. Eine ganz andersartig gelagerte Aufgabe dabei war der Ausbau eines Teils der historischen Festungsanlage der Stadt Torgau zur „Kulturbastion“ - ein soziokulturelles Zentrum. Ziel war es, in den Tonnen der Bastion einen Treffpunkt für vielfältige Aktivitäten zu schaffen. Dabei war es gelungen, Mittel der EU, des Landes und des Bundes, der Stadt Torgau und des Leipziger Kulturraumes zu verbinden. Trägerverein ist KAP Torgau e.V., welcher eine offene Jugendarbeit mit vielfältigen kulturellen Veranstaltungen für jede Altersgruppe verbindet. Das Soziokulturelle Zentrum Kulturbastion Torgau wurde mit dem Kulturpreis der Kulturpolitischen Gesellschaft ausgezeichnet und ist heute fester Bestandteil der Kulturlandschaft der Stadt.

Das Spektrum der Projekte war noch vielfältiger und reichte weiter von der Unterstützung des kleinen Einzelhandels, des Ausbaus der innerstädtischen Verkehrsinfrastruktur bis hin zur Schwimmhallensanierung.

Die Möglichkeit, Mittel zu kumulieren, versetzte die Stadt in die Lage, im Programmgebiet 27 Einzelprojekte mit Gesamtausgaben in Höhe von ca. 11,8 Millionen Euro erfolgreich umzusetzen. Dabei konnte der Eigenanteil der Stadt in Höhe von ca. 1,37 Millionen Euro teilweise durch andere Mittel (unter anderem Kulturraum, Städtebauförderung, Denkmalfördermittel, Bundesmittel für Kultur) ersetzt werden. 
Die Torgauer Museumslandschaft wurde zwischenzeitlich um weitere Objekte erweitert. Mit Hilfe von Städtebaufördermitteln, Stiftungsgeldern und mit viel Engagement des Fördervereins Denkmalpflege Torgau wurden ein kleines Bürgerhaus am Rosa-Luxemburg-Platz und das Spalatinhaus in der Katharinenstraße restauriert. Im Ergebnis konnte in der Altstadt erstmals 2009 die negative Tendenz der Einwohnerentwicklung umgekehrt werden, so dass wieder mehr als 5.000 Einwohner hier lebten. Dennoch ist nicht zu verkennen, dass der Leerstand in der Altstadt (Wohnen und Gewerbe) den höchsten Stand innerhalb der Gesamtstadt aufzuweisen hat.

Weiterhin von Bedeutung sind die Städtebaufördermaßnahmen im Rahmen der Stadterneuerung. Bereits 1992 wurde das Erhaltungssatzungsgebiet „Historische Altstadt“ (126 Hektar) beschlossen. Hauptanliegen ist der Schutz der städtebaulichen Eigenart, der ortsüblichen Bauarchitektur sowie der Milieuschutz. Ergänzt wurden die Zielstellungen zur Entwicklung der Innenstadt im Rahmen der Sanierungssatzung für das Gebiet „Altstadtkern I“ (30 Hektar). Auf dieser Grundlage wurde Torgau bereits kurz nach der Wende in die Programme „Städtebaulicher Denkmalschutz“ (SDP) und „Städtebauliche Erneuerung“ (SEP) aufgenommen. Unter Beachtung der unterschiedlichen Förderbedingungen war so eine Konzentration der Fördermittel auf das Stadtzentrum zielführend.

Schwerpunkte in den letzten Jahren waren die umfangreichen Umgestaltungsmaßnahmen im Schloss Hartenfels zur 2. Sächsischen Landesausstellung 2004, die Sanierung des Ausstellungsflügels (Flügel D), die Neugestaltung des Rosengartens und des Schlosshofes und ab 2010 die umfangreichen Vorbereitungen des Lutherjubiläums 2017. Dies betraf insbesondere die Fertigstellung des Flügels E (Innenausbau), die Sanierung des Bärengrabens mit der eingebundenen Stadtmauer und der Fassaden der Flügel A und $B$.

Städtische Vorhaben wie die Errichtung der Stadtbibliothek Ritterstraße 10 und des Stadtund Kulturgeschichtlichen Museums in der Wintergrüne 5, die Errichtung des Seniorenselbsthilfezentrums im Fleischmarkt, die Sanierung der Mittelschule „Katharina von Bora“, die Restaurierung von Teilen des Rathauskomplexes sowie von Häusern der Museumslandschaft, so zum Beispiel Breite Straße 9, Pfarrstraße 3, Fischerstraße 11, Rosa-Luxemburg-Platz 4 und die Katharinenstraße 10, wurden seither umgesetzt. Erwähnenswert sind weiterhin die Instandsetzung der Türme und die Freiflächengestaltung um die Marienkirche, die Neugestaltung des Alltagskirchenvorplatzes, die Sanierung der mittelalterlichen Stadtmauer und einzelner innerstädtischer Straßenräume, wie zum Beispiel Bäckerstraße, Breite Straße, Ritterstraße, Scheffelstraße und Fischerstraße, sowie zahlreiche Sanierungsmaßnahmen in privaten Grundstücken. Insgesamt wurden mehr als 220 private Bauvorhaben unterstützt.

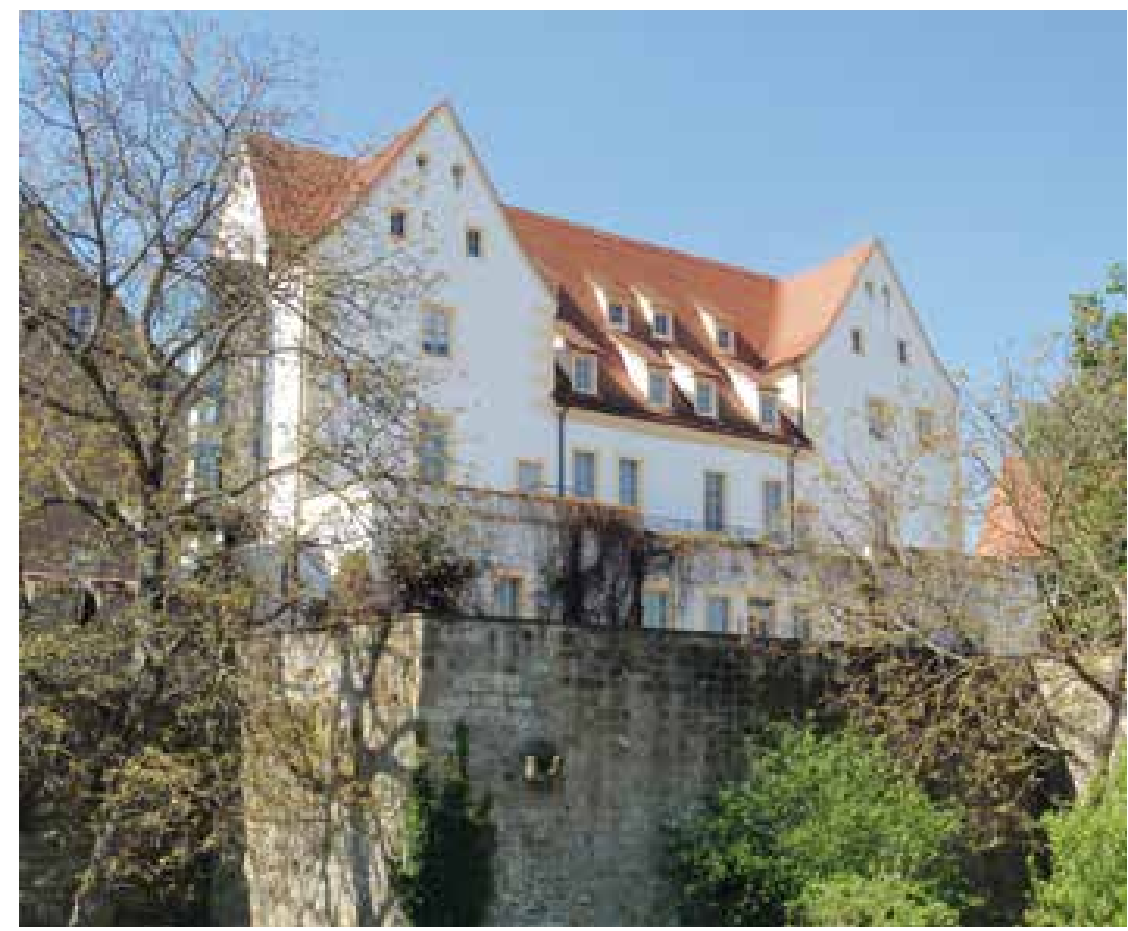

Mit der Programmausschreibung 2014 erhielt Torgau die Möglichkeit eine Antragstellung zur Neuaufnahme in die 2. Förderperiode des Städtebaulichen Denkmalschutzes (SDP (N)). Durch die Verkleinerung des ursprünglichen Fördergebietes in der Altstadt erfolgte im Einklang mit der gesamtstädtischen Entwicklung (Leitlinie: Innenentwicklung vor Außenentwicklung) eine Konzentration der Förderung. Das neue Fördergebiet „Altstadt Torgau“ mit einer Größe von ca. 70 Hektar umfasst neben dem Altstadtkern auch Teile des Areals der zukünftigen Landesgartenschau 2022 in Torgau. Schwerpunkt des Förderprogramms ist gegenwärtig die Errichtung einer Jugendherberge in unmittelbarer Nachbarschaft zum Schloss Hartenfels. Die Stadt möchte ab 2019 mit der Bereitstellung kostengünstiger Unterkünfte verstärkt Fami-

Torgau, Stadt- und Kulturgeschichtliches Museum in der früheren Kurfürstlichen Kanzlei nach der Sanierung ๑) Stadtverwaltung Torgau
Marktplatz und Rathaus nach der Sanierung ๑ Stadtverwaltung Torgau

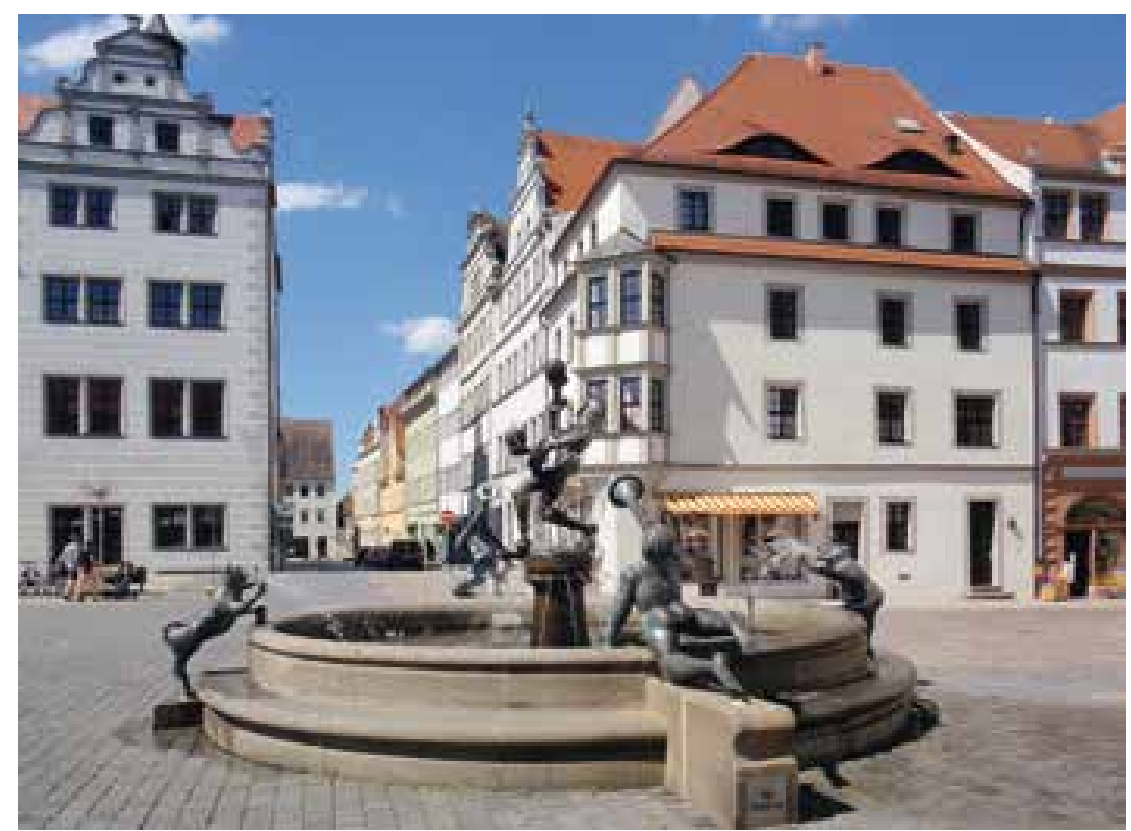



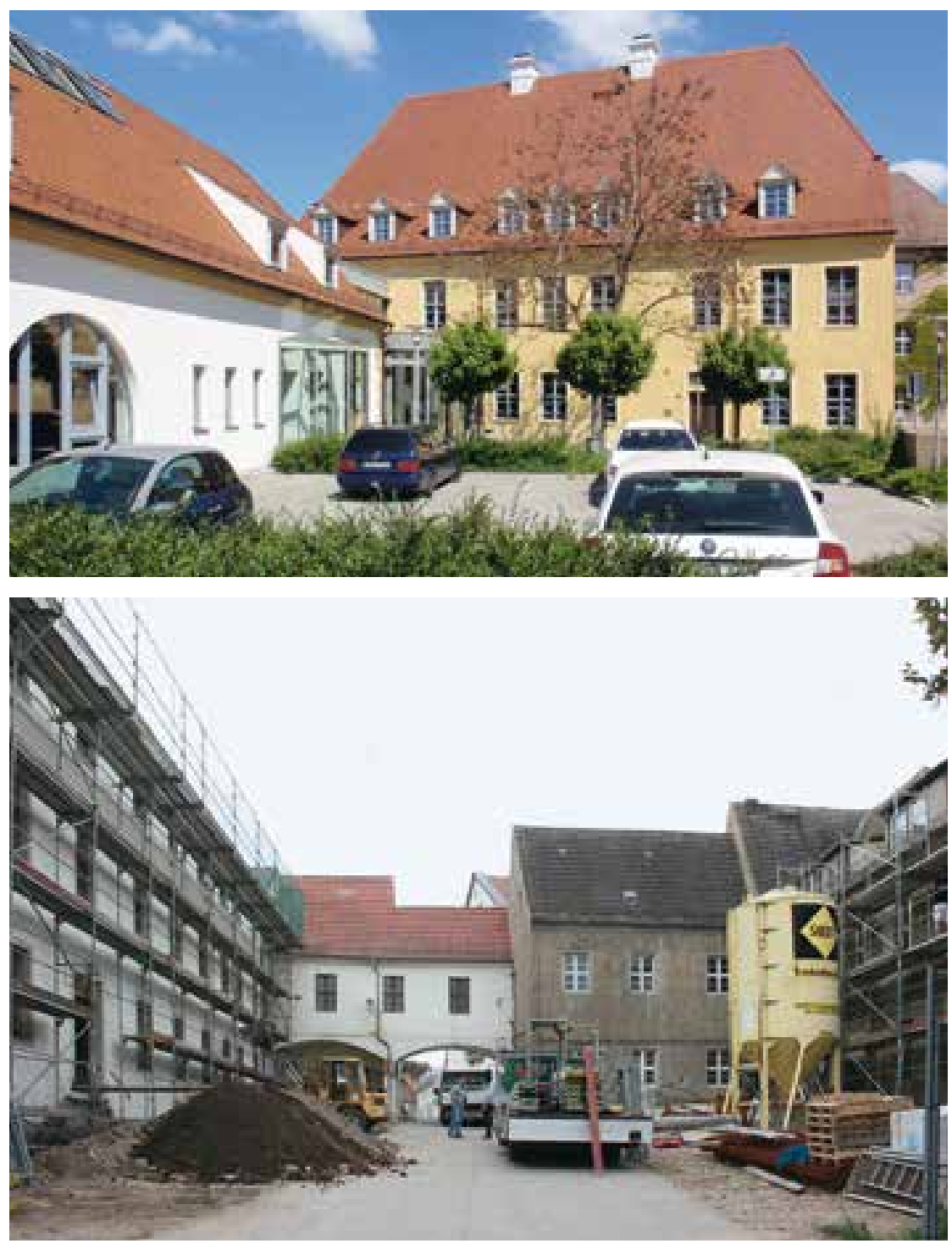

oben links: Torgau, sanierte Stadtbibliothek

- Stadtverwaltung Torgau

oben rechts: Torgau, „Junger Garten", noch zu entwickelndes Gelände der Landesgartenschau

2022

- Stadtverwaltung Torgau

unten: Torgau, Bau der Jugendherberge, 2017

๑ Stadtverwaltung Torgau

\section{Autoren}

Dagmar Rausch 1990-2015 Planungsamtsleiterin

Karin Hahn

Mitarbeiterin im Planungsamt, Sanierungsberatung lien mit Kindern und Jugendliche als Touristen begrüßen. Als Fertigstellungstermin ist gegenwärtig Herbst 2019 geplant.

Insgesamt wurden für die Sanierung in den Fördergebieten der Altstadt seit 1991 mehr als 66 Millionen Euro investiert. Dabei stellten Bund und Land über 44 Millionen Euro zur Verfügung. Der dafür aufzubringende Eigenanteil durch die Stadt Torgau betrug 12 Millionen Euro.

Im September 2017 wurde ein weiteres Städtebaufördergebiet unter der Bezeichnung „Torgau Nördlicher Stadtpark“ beschlossen. In diesem Gebiet sollen in den nächsten Jahren städtebauliche Maßnahmen umgesetzt werden, die insbesondere die Verbesserung der urbanen grünen Infrastruktur, des Stadtklimas und der Umweltgerechtigkeit zum Ziel haben.

\section{Sächsische Landesgartenschau 2022}

2016 bewarb sich die Stadt Torgau mit der Konzeption „Natur. Mensch. Geschichte - Grüne Renaissancestadt Torgau“ um die Ausrichtung der 9. Sächsischen Landesgartenschau 2022 und erhielt den Zuschlag für die Ausrichtung.

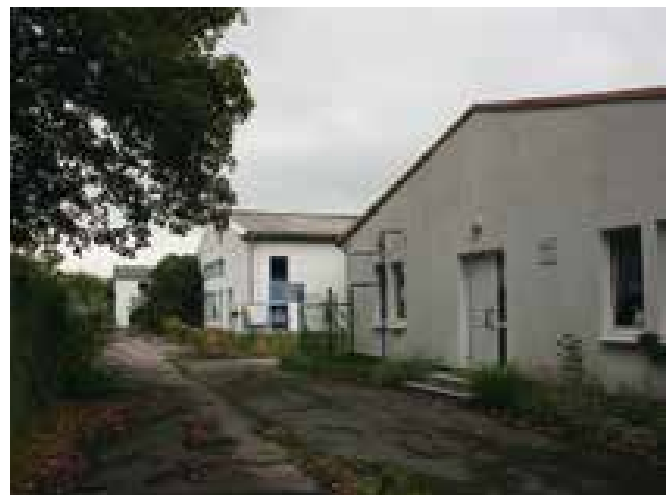

Die Gartenschau kann die Stadt, die Region, ihre Menschen, ihre Gärten und ihre Natur überregional bekannter machen und das Image der Stadt um eine neue „grüne Facette“ erweitern. Schwerpunkte sind die Sanierung und Aufwertung des von hoher ökologischer Bedeutung geprägten Gartendenkmals (Glacis), die Sanierung der integrierten Teiche sowie der Erhalt der biologischen Vielfalt und Naturerfahrung.

Im nördlich daran angrenzenden Bereich, dem „Jungen Garten“ sollen durch die Schaffung eines zusätzlichen Erlebnis- und Naherholungsortes sowie der Verbesserung der vorhandenen Kleingartenstruktur Impulse für eine dauerhafte und umwelt- bzw. ressourcenschonende Entwicklung der Stadt gegeben werden. Zur Umsetzung der einzelnen Ideen und Maßnahmen ist die aktive Beteiligung der Bürgerinnen und Bürger, insbesondere aber die Mitarbeit der Kinder und Jugendlichen, aber auch der älteren Generation von großer Bedeutung.

\section{Fazit}

Die Anstrengungen zur Erhaltung unserer Stadt wurden genutzt und mit Engagement in den vergangenen Jahren zahlreiche anspruchsvolle Projekte umgesetzt. Das Stadtbild präsentiert sich zur Überraschung und Freude der Besucher weiterhin als geschlossenes wertvolles Renaissanceensemble. Trotz der zahlreichen bisher umgesetzten Maßnahmen unter Zuhilfenahme der Bund-Länder-Programme der Städtebauförderung, der EU-Förderung und des Einsatzes von kommunalen und privaten Mitteln konnten im Stadtgebiet bisher nicht alle Missstände beseitigt und Vorhaben umgesetzt werden. Der große Anteil historischer Bebauung bringt auch in Zukunft eine große Herausforderung mit sich.

Die Stadtentwicklung ist als langfristiger Prozess zu verstehen, bei dem die Interessen und Möglichkeiten der verschiedenen Akteure (Eigentümer, Gewerbetreibende, Mieter, Stadt, Wohnungsunternehmen, Denkmalpflege usw.) berücksichtigt werden müssen.

Die Bürger der Stadt Torgau und insbesondere die Grundstückseigentümer sind die Hauptbeteiligten an diesem Prozess. Ohne deren Bereitschaft zur Mitwirkung haben Stadtumbau und Stadtsanierung keinen Erfolg. 